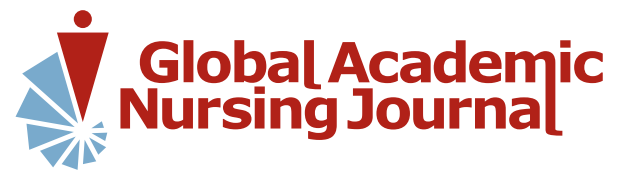

\section{Violência sexual masculina: uma revisão integrativa}

Male sexual violence: an integrative review

Violencia sexual masculina: una revisión integradora

\section{Carolina Pimentel Machado ${ }^{1}$ ORCID: 0000-0001-6883-8967}

Adriana Elisa da Silva Santana ${ }^{2}$ ORCID: 0000-0001-9809-0259

Aluísio Gomes da Silva Júnior ${ }^{3}$ ORCID: 0000-0003-2445-3963

${ }^{1}$ Universidade Veiga de Almeida. Rio de Janeiro, Brasil.

${ }^{2}$ Rio Saúde. Rio de Janeiro, Brasil. ${ }^{3}$ Instituto de Saúde Coletiva. Bahia, Brasil.

\section{Como citar este artigo:}

Machado CP, Santana AES, Júnior AGS. Violência sexual masculina: uma revisão integrativa. Glob Acad Nurs. 2021;2(Sup.2):e177. https://dx.doi.org/10.5935/26755602.20200177

\section{Autor correspondente:}

Carolina Pimentel Machado

E-mail: carolina.machado@uva.br

Editor Chefe: Caroliny dos Santos Guimarães da Fonseca Editor Executivo: Kátia dos Santos Armada de Oliveira

Submissão: 24-06-2021

Aprovação: 20-07-2021

\section{Resumo}

Objetivou-se identificar os estudos realizados sobre violência sexual, tendo como vítimas pessoas do sexo masculino, sendo realizada uma revisão integrativa. Os dados foram coletados na Biblioteca Virtual de Saúde, Literatura Latino Americana e do Caribe em Ciências da Saúde, Literatura Internacional em Ciências da Saúde, Biblioteca Eletrônica Científica On-line e Banco de Dados de Enfermagem. A amostra contou com três publicações no idioma inglês, após a aplicação dos descritores utilizados. Identificou-se que é deficiente o atendimento às vítimas de violência sexual do sexo masculino, comparando-se com as do sexo feminino. O conhecimento deste cenário é importante, no sentido de que as equipes de saúde, especialmente os enfermeiros, sejam melhores preparados do ponto de vista técnico e emocional para lidar com este tipo de situação.

Descritores: Atenção à Saúde; Cuidados de Enfermagem; Enfermagem; Saúde do Homem; Delitos Sexuais.

\section{Abstract}

The aim was to identify the studies carried out on sexual violence, with male victims, and an integrative review was carried out. Data were collected from the Virtual Health Library, Latin American and Caribbean Health Sciences Literature, International Health Sciences Literature, Online Scientific Electronic Library and Nursing Database. The sample had three publications in the English language, after applying the descriptors used. It was identified that care for male victims of sexual violence is deficient, compared to female victims. Knowledge of this scenario is important, in the sense that health teams, especially nurses, are better prepared from a technical and emotional point of view to deal with this type of situation.

Descriptors: Delivery of Health Care; Nursing Care; Nursing; Men's Health; Sex Offenses.

\section{Resumén}

El objetivo fue identificar los estudios realizados sobre violencia sexual, con víctimas masculinas, y se realizó una revisión integradora. Los datos se obtuvieron de la Biblioteca Virtual en Salud, la Literatura de Ciencias de la Salud de América Latina y el Caribe, la Literatura de Ciencias de la Salud Internacional, la Biblioteca Electrónica Científica en Línea y la Base de Datos de Enfermería. La muestra incluyó tres publicaciones en idioma inglés, luego de aplicar los descriptores utilizados. Se identificó que la atención a los hombres víctimas de violencia sexual es deficiente, en comparación con las mujeres víctimas. El conocimiento de este escenario es importante, en el sentido de que los equipos de salud, especialmente las enfermeras, están mejor preparados desde el punto de vista técnico y emocional para enfrentar este tipo de situaciones.

Descriptores: Atención a la Salud; Atención de Enfermería; Enfermería; Salud del Hombre; Delitos Sexuales. 


\section{Introdução}

A Lei n.o 12.015/2009 modificou o Título VI da Parte Especial do Decreto Lei n. 2.848/1940 - Código Penal e também o Art. 10 da Lei n.o 8.072/1990, que trata sobre crimes hediondos, incluindo como crimes sexuais, as situações que atentem contra a dignidade sexual, a liberdade sexual e criando o conceito de vulnerabilidade sexual, além de considerar crime, qualquer forma de exploração sexual ${ }^{1-3}$.

Neste contexto o estupro passou a ser enquadrado no Art. 213 do Código Penal Brasileiro com uma nova redação: "Constranger alguém, mediante violência ou grave ameaça, a ter conjunção carnal ou a praticar ou ainda permitir que com ele se pratique outro ato libidinoso" ${ }^{2}$.

Violência sexual é definida como: "todo ato sexual, tentativa de consumar um ato sexual ou insinuações sexuais indesejadas, ou ações para comercializar ou usar de qualquer outro modo a sexualidade de uma pessoa por meio da coerção por outra pessoa, independentemente da relação desta com a vítima, em qualquer âmbito, incluindo o lar e o local de trabalho"4.

Estudos indicam um movimento geral na direção de que a violência sexual do ponto de vista da saúde pública resulta da combinação de diversos fatores de risco em nível individual, comunitário e social e o combate à violência sexual requer o envolvimento de vários setores, sendo eles: saúde, educação, assistência social e justiça criminal ${ }^{4}$.

As consequências advindas de um ato de violência sexual são difíceis de mensuração, e afetam diretamente além das vítimas, seus familiares. No âmbito da saúde do violentado os danos causados são expressivos e diversas vezes irreparáveis. Cabe ressaltar que grande parte dos sobreviventes acabam sendo infectados por IST/Aids, impactando diretamente seu estado emocional e físico.

Os casos de violência sexuais levadas aos Hospitais da Rede Pública devem ser encaminhados a equipes bem preparadas tanto do ponto de vista psicológico quanto do técnico para que seja oferecido um atendimento eficiente e adequado.

Dentre os profissionais envolvidos no atendimento às vítimas destaca-se o papel do enfermeiro, que deve estar atento para identificar os sinais e sintomas de um ato de violência sexual e atuar de acordo com o protocolo de atendimento da Secretaria de Saúde do Paraná que é uma das referências em atendimento no País.

Segundo esse protocolo, a primeira etapa e de fundamental importância é o acolhimento que deve ser realizado com ética, privacidade, confidencialidade e sigilo. O enfermeiro deve confirmar o tempo decorrido entre a violência sexual e a procura pelo atendimento e se o agressor utilizou ou não preservativo ${ }^{5}$.

As informações preliminares colhidas deverão ser registradas em prontuário para que a vítima não repita várias vezes o fato ocorrido, em seguida o atendimento clínico é efetuado pelo médico. Casos de violência sexual devem ser notificados imediatamente (24 horas) para a Secretaria Municipal de Saúde ${ }^{5}$.
Como a violência sexual é um problema também de saúde pública, o Estado tem por obrigação garantir às vítimas de violência sexual o acesso a serviços e apoio adequados $^{4}$.

Esta pesquisa partiu do pressuposto de que a problemática da violência sexual envolvendo vítimas do sexo masculino é ainda pouco explorada e abordada pela comunidade acadêmica, o que contribui para o desconhecimento e despreparo do enfermeiro por ocasião do atendimento a estas vítimas. O objetivo deste artigo foi identificar estudos realizados sobre violência sexual envolvendo vítimas do sexo masculino através de uma pesquisa integrativa em artigos nacionais e internacionais (idioma inglês), utilizando descritores previamente definidos e a partir do resultado encontrado propor ações visando a melhoria do conhecimento do Enfermeiro frente ao atendimento de vítimas de violência sexual do sexo masculino.

\section{Metodologia}

Este estudo baseou-se em uma revisão integrativa. A revisão integrativa de literatura, pode ser definida como: um método que têm por característica uma ampla abordagem metodológica das revisões que permite incluir múltiplos estudos com diversos delineamentos de pesquisa para compreender o fenômeno estudado, podendo combinar tanto dados teóricos quanto empíricos, incluindo definições, conceitos, teorias, além de análise de problemas metodológicos de um tópico em particular ${ }^{6}$.

O método de revisão integrativa pode ser utilizado em pesquisas realizadas em outras áreas, além das áreas de saúde e educação, pois viabiliza a capacidade de conhecimento científico fazendo com que o pesquisador possa se aproximar da problemática a ser explorada, tendo possibilidade de visualizar a produção científica já realizada e também a própria evolução do tema ao longo do tempo e, verificar possíveis oportunidades de pesquisa ${ }^{7}$.

Portanto, de acordo com esses autores, essa técnica deve ser adotada quando se pretende realizar uma análise e síntese do conhecimento científico já produzido acerca do tema investigado. $O$ método obedeceu às seguintes etapas: a criação da pergunta norteadora, a definição dos descritores, a busca nas bases de dados especializadas, a tabulação de resultados e análise.

Com os descritores selecionados, procedeu-se à associação de filtros disponíveis na base de dados especializados e objetivando realizar uma busca mais precisa, foram estabelecidos os seguintes critérios de inclusão: textos completos disponíveis, idiomas português e inglês e que tenham como período de publicação os anos de 2013 a 2018. Como critérios de exclusão foram descartados artigos que fugiram da temática e do contexto, duplicados e incompletos. Essa busca foi feita entre o período de 4 de dezembro de 2018 a 4 de janeiro de 2019.

O Fluxograma a seguir Figura 1, demonstra o processo de seleção dos artigos de maneira objetiva e resumida desta revisão integrativa. 


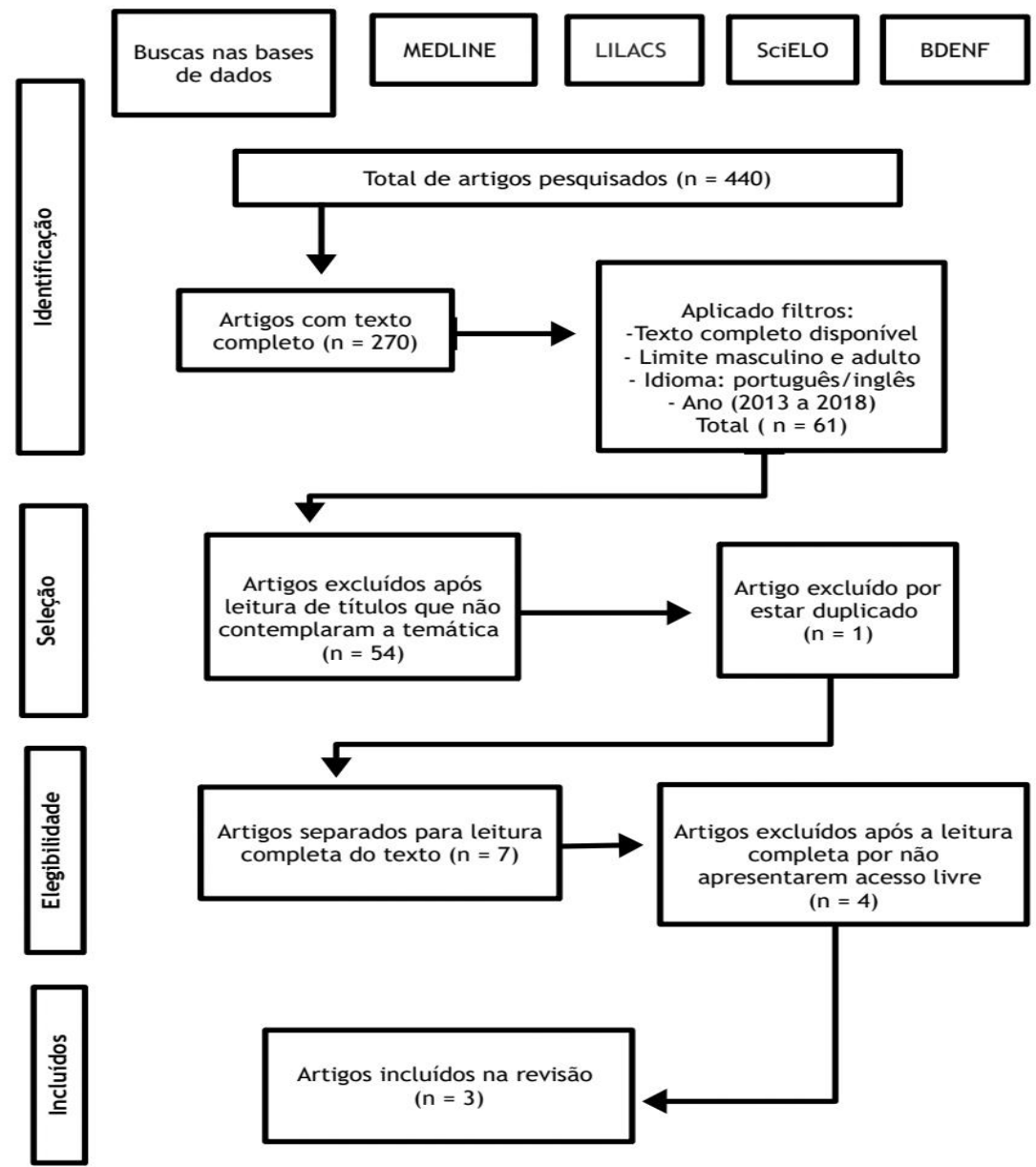

A pergunta norteadora foi elaborada por meio da estratégia $\mathrm{P}=$ População, $\mathrm{I}=$ Interesse, $\mathrm{Co}=$ Contexto $(\mathrm{PICO})$ : Os enfermeiros estão capacitados ao atendimento de pacientes do sexo masculino violentados sexualmente?

Os descritores utilizados foram definidos por meio da ferramenta Descritores em Ciência e Saúde (DeCS), totalizando 3 descritores selecionados, sendo eles: Saúde do homem, Enfermagem e violência sexual.

O levantamento bibliográfico eletrônico foi feito nas bases contidas na Biblioteca Virtual em Saúde (BVS), Literatura Latino Americana e do Caribe em Ciências da Saúde (LILACS), Literatura Internacional em Ciências da
Saúde (MedLine), Scientific Eletronic Library Online (SciELO) e Banco de Dados de Enfermagem (BDENF).

\section{Resultados}

Os resultados e as análises das publicações selecionadas como: título, ano, idioma, bibliografia, base de dados e objetivo do trabalho, são apresentados no Quadro 1 abaixo. A evidência e grau de recomendação também constantes no Quadro estão de acordo com modelo Oxford Centre for Evidence-Based Medicine.

Quadro 1. Publicações localizadas nas bases de dados sobre violência sexual e saúde do homem segundo título, ano, idioma, bibliografia, base de dados, objetivo, evidência e grau de recomendação. Rio de Janeiro, RJ, Brasil, 2019

\begin{tabular}{|c|c|c|c|c|}
\hline Título & $\begin{array}{c}\text { Ano de } \\
\text { Publicação }\end{array}$ & Idioma & Base de dados & $\begin{array}{c}\text { Evidência e } \\
\text { Grau de } \\
\text { Recomendação }\end{array}$ \\
\hline The male victim of sexual assault & 2013 & Inglês & MedLine & $\begin{array}{c}\text { Auxiliar os profissionais de saúde } \\
\text { no atendimento ao homem } \\
\text { vítima de violência sexual. }\end{array}$ \\
\hline $\begin{array}{c}\text { Sexual violence against men and boys } \\
\text { in conflict and forced displacement: } \\
\text { implications for the health sector }\end{array}$ & 2017 & Inglês & MedLine & $\begin{array}{c}\text { Descrever os desafios e sugerir } \\
\text { melhorias na prestação da } \\
\text { assistência em saúde às vítimas } \\
\text { de violência sexual do sexo } \\
\text { masculino. }\end{array}$ \\
\hline
\end{tabular}


A totalidade dessa pesquisa trata do homem vítima de violência sexual, apontando estatísticas, pontos de vista da sociedade, as consequências psicológicas e os serviços de saúde colocados à disposição das vítimas ${ }^{8}$.

Estudos realizados e catalogados em países ocidentais como Reino Unido, EUA e países nórdicos apresentaram que em média $5 \%$ a $10 \%$ dos casos de estupro registrados têm como vítimas pessoas do sexo masculino ${ }^{8}$.

Os artigos números 2 e 3 da Quadro 1 abordam mais especificamente casos de violência sexual em conflitos envolvendo homens e reconhecem que casos desta natureza vêm aumentando gradualmente, embora meninas e mulheres sejam o grupo ainda mais afetado. Ocorreu em 2013, uma grande mudança no sentido da inclusão de vítimas do sexo masculino nas políticas internacionais sobre violência sexual em tempo de guerra. O Conselho de Segurança da ONU documentou um alto índice de violência sexual contra homens, incluindo estupro, atos sexuais forçados, além da castração no conflito da ex-lugoslávia. No Oriente Médio, refugiados compartilharam histórias de violência sexual, incluindo tortura e estupro contra homens, principalmente na Síria. Dentro deste contexto os agentes de saúde humanitários não sabiam como lidar com este tipo de situação, não sabiam onde encaminhá-los para cuidados médicos e serviços psicossociais. Uma gestora de programas de violência baseada em gênero no Iraque descreveu que médicos e enfermeiras durante um treinamento sobre cuidados pós-estupro comentavam e se surpreendiam com a situação de homens vítimas de estupro ${ }^{8,9}$.

De acordo com o Centro de Referência de Violência Sexual de St Mary's Hospital em Manchester, Reino Unido, apesar de ser um grupo bem menor quando comparado com vítimas de violência sexual do sexo feminino, os casos de violência sexual envolvendo homens é o que mais têm crescido. No leste da República Democrática do Congo, uma pesquisa de base populacional descobriu que quase um quarto dos homens $(23,6 \%)$ havia sofrido violência sexual. Uma outra pesquisa feita pela Organização Médicos Sem Fronteiras concluiu que de 429 refugiados que fugiram da violência na América Central, $17 \%$ dos homens relataram ter sofrido abuso sexual enquanto estavam em trânsito pelo México ${ }^{8,9}$.

\section{Discussão}

A sociedade em geral mantém o entendimento de que a violência sexual envolvendo homens são casos raros, pois acreditam que estes são capazes de resistir e que esses episódios ocorrem em prisões, além disso, entende que os homens devem ser fortes o suficiente para lidar com essa situação. Comparando-se particularmente o estupro entre vítimas do sexo masculino e feminino percebe-se que quanto mais forte é o estereótipo de estupro masculino temos uma culpabilidade maior do homem vítima de tal crime, ao mesmo tempo em que a culpa atribuída ao estuprador é reduzida. Sendo assim, percepções de masculinidade são, portanto, mais influentes quando se considera estupro masculino do que as percepções de feminilidade sobre estupro masculino-feminino ${ }^{10}$.

Em geral, as vítimas do sexo masculino sentem-se mais culpadas do que as vítimas do sexo feminino, isso faz com que os homens sofram por sentir que sua masculinidade foi diminuída perante a sociedade ${ }^{10}$.

Foi constatada também a escassez de serviços de saúde voltados para o atendimento aos homens vítimas de violência sexual quando comparados com os serviços à disposição de pessoas do sexo feminino, além da existência de barreiras ao acesso a esses cuidados. São raros também os protocolos de atendimento para casos de violência sexual envolvendo homens. É imperativa a necessidade de orientações específicas às equipes de saúde sobre como se prestar assistência às vítimas de violência sexual do sexo masculino e desfazer mitos sobre a vitimização sexual masculina ${ }^{8,10}$

No Reino Unido foram criados Centros de Referência para tratamento de vítimas de violência sexual, mas após 25 anos da criação destes Centros em Manchester, o que se vê são atendimentos às vítimas mulheres na sua esmagadora maioria em detrimento dos homens, o que retrata ainda a existência de barreiras para o tratamento do homem vítima de violência sexual ${ }^{9}$.

Um fato relevante é que os autores da violência sexual, cujas vítimas são homens utilizam na maioria das vezes objetos para realizar a penetração e que a violação peniana está significativamente presente quando as vítimas se tratam de mulheres. Os homens geralmente são violentados por duas ou mais pessoas, sugerindo-se assim uma constância de "estupros coletivos"8,10.

O efeito psicológico é profundo em casos de violência e em especial os de natureza sexual, produzindo consequências desestabilizadoras e multidimensionais, como depressão, ansiedade, transtorno de estresse póstraumático e ideação suicida. Dados do St Mary's Center apontam que vítimas do sexo masculino são mais propensas a tentar negar o caso de violência sofrido. Contribui para esta afirmação o percentual de $41,6 \%$ de mulheres vítimas que retornam para realizar tratamento, enquanto o percentual fica em $27,6 \%$ quando se trata de homens. 0 trauma psicológico nos homens se dá principalmente pela ideia do conflito com o papel sexual dominante e a presunção de que pessoas do sexo masculino são suficientemente agressivas e fortes, podendo evitar qualquer contato de natureza sexual com outros homens. $\mathrm{O}$ estudo descobriu que as vítimas do sexo masculino apresentaram níveis significativamente mais altos de sofrimento comparando-se com vítimas do sexo feminino, devido a uma variedade de fatores específicos do contexto e da cultura, incluindo a vergonha, medo da descoberta pela comunidade e do estigma social resultante, além do medo de represálias. Danos para a saúde das vítimas são 
apresentados principalmente através de doenças sexualmente transmissíveis incluindo HIV, incontinência, comprometimento genital e retal, infertilidade e disfunção sexual ${ }^{8,10}$.

Um outro estudo realizado em Londres, envolvendo 115 homens, em um serviço de tratamento psicológico, constatou-se que o tempo decorrido entre a prática da violência sexual e o atendimento foi de pelo menos 16 $\operatorname{anos}^{10}$.

No plano internacional existe um outro obstáculo a ser considerado e que contribui para que os homens fiquem desencorajados a denunciar casos de violência sexual sofridas é que em pelo menos 79 países, a sodomia é considerada crime, além disso diversas leis nestes mesmos países não consideram as vítimas de violência sexual do sexo masculino em suas definições de estupro. A polícia classifica casos de violência sexual envolvendo homens como um abuso ou um dano corporal geral, processado no máximo como tortura ou espancamento ${ }^{9}$.

\section{Conclusão}

Como podemos ver a partir da discussão dos resultados, a violência sexual masculina é um tema pouco abordado no Brasil e que nenhum estudo encontrado tratou especificamente da capacitação do enfermeiro para o atendimento a clientes homens vítimas de violência sexual. Buscou-se então, estudos envolvendo este tema no âmbito internacional que embora também sejam escassos, forneceu
3 (três) artigos que foram utilizados como fontes deste estudo.

Existe a necessidade de que estudos sobre violência sexual contra pessoas do sexo masculino sejam produzidos em âmbito nacional. Quanto maior for o número de estudos disponíveis, melhor será o atendimento realizado pelo enfermeiro e demais profissionais de saúde envolvidos no atendimento. Pessoas próximas às vítimas devem incentivar o registro e encaminhamento aos órgãos competentes.

Foi constatado que alguns fatores contribuem para a escassez de estudos relativos ao tema, destacando-se: a dificuldade de comunicação dos homens para relatarem este tipo de violência, o medo de sofrer um prejulgamento pela sociedade e a própria vergonha pela situação vivida.

É fato que casos de violência sexual envolvendo homens são em menor número, comparando-se com casos envolvendo mulheres, porém precisam do mesmo nível de atenção e de importância pelas autoridades. Não devemos ver o homem somente como o agressor e sim como vítima também.

Treinamentos e um programa de capacitação do enfermeiro devem ser implantados no âmbito das Secretarias de Saúde do País para que aconteça um atendimento eficiente a essas vítimas.

O artigo buscou alertar para a importante necessidade de produção de literatura nacional sobre a violência sexual envolvendo vítimas do sexo masculino que proporcionará um conhecimento mais sólido e concreto a respeito dos aspectos e particularidades desta problemática.

\section{Referências}

1. Brasil. Lei n.o 12.015, de 7 de agosto de 2009. Altera o Título IV da Parte especial do Decreto Lei n.o 2.848 , de 7 de dezembro de 1940 Código Penal que dispõe sobre crimes hediondos [Internet]. Brasília (DF): Brasil; 2009 [acesso em 19 dez 2018]. Disponível em: http://www.planalto.gov.br/ccivil_03/_ato2007-2010/2009/lei/l12015.htm

2. Brasil. Lei n.o 2.848/1940, de 7 de dezembro de 1940. Código Penal [Internet]. Brasília (DF): Brasil; 1940 [acesso em 19 dez 2018 ]. Disponível em: https://www2.camara.leg.br/legin/fed/declei/1940-1949/decreto-lei-2848-7-dezembro-1940-412868-publicacaooriginal1-pe.html

3. Brasil. Lei n.o 8.072, de 25 de julho de 1990. Dispõe sobre os crimes hediondos, nos termos do art. 5o, inciso XLIII, da Constituição Federal, e determina outras providências [Internet]. Brasília (DF): Brasil; 1990 [acesso em 19 dez 2018]. Disponível em: http://www.planalto.gov.br/ccivil_03/leis/l8072.htm

4. Organização Pan Americana da Saúde (OPAS). Organização Mundial da Saúde (OMS). Neste Dia Laranja, OPAS/OMS aborda violência sexual e suas consequências para as vítimas [Internet]. Brasília (DF): OPAS/OMS; 2018 [acesso em 19 Dez 2018]. Disponível em: https://www.paho.org/pt/noticias/25-7-2018-neste-dia-laranja-opasoms-aborda-violencia-sexual-e-suas-consequencias-para

5. Secretaria de Estado de Saúde (SES). Protocolo para atendimento às pessoas em situação de violência sexual [Internet]. Curitiba (PR): SES; 2017 [acesso em 16 mai 2019]. Disponível em: https://criminal.mppr.mp.br/arquivos/File/ProtocoloViolenciaSexualSESA.pdf

6. Souza MT, Silva MD, Carvalho R. Revisão integrativa: o que é e como fazer. Einstein. 2010;8(1). DOI: 10.1590/S1679-45082010RW1134

7. Botelho LLR, Cunha CCA, Macedo M. O método da revisão integrativa nos estudos organizacionais. GeS [Internet]. 2011 [acesso em 19 jan 2019];5(11):121-136. Disponível em: http://www.gestaoesociedade.org/gestaoesociedade/article/view/1220/906

8. Chynoweth SK, Freccero J, Touquet $\mathrm{H}$. Sexual violence against men and boys in conflict and forced displacement: implications for the health sector. Reprod Health Matters. 2017;25(51):90-94. DOI: 10.1080/09688080.2017.1401895

9. Touquet $\mathrm{H}$, Gorris E. Out of the shadows? The inclusion of men and boys in conceptualisations of wartime sexual violence. Reprod Health Matters. 2016;24(47):36-46. DOI: 10.1016/j.rhm.2016.04.007

10. Mclean IA. The male victim of sexual assault. Best Practice \& Research Clinical Obstetrics and Gynaecology. 2013;27(1):39-46. DOI: 10.1016/j.bpobgyn.2012.08.006 